\title{
Neutrophil-Lymphocyte count ratio on admission as a predictor of Bacteremia and In Hospital Mortality among Sepsis and Septic shock In Patients at Rizal Medical Center
}

\author{
Rakesh Kumar Mandal' ${ }^{1}$, Primo B. Valenzuela ${ }^{2}$ \\ ${ }^{1}$ Resident Physician, ${ }^{2}$ Medical Specialist, Department of Internal Medicine, Rizal Medical Center, Pasig Blvd., Pasig \\ City, Philippines, 1600
}

Background: Neutrophil-Lymphocyte count ratio (NLCR), a readily accessible biomarker, has become increasingly useful as predictive and prognostic tools in patients with various medical conditions. NLCR has also been reported to represent disease severity effectively. Aims and Objectives: To investigate NLCR on admission as a prognostic marker of bacteremia and inhospital mortality in patients diagnosed with Sepsis and Septic Shock. Materials and Methods: This cross-sectional, retrospective study involves one-hundred twenty (120) adult patients with Sepsis and Septic Shock admitted to the IMCU and ICU from January 2015 to December 2016. Laboratory data and clinical outcomes were retrieved and documented from the laboratory and patients' charts. A cut-off point of $>10$ was used to be a marker for bacteremia and hence, patients were stratified into two groups based on this cut-off. Out of 120, 78 patients (with mean age of 60 ) has NLCR of at least 10 while only 42 patients (with mean age of 55) has NCLR of at most 10. Results: A significant, strong and positive association between NLCR and in-hospital mortality was found $(P=0.0001)$ indicating that an increased rate of mortality is observed for patients with persistently high NCLR. Results also indicated that NLCR is a significant predictor $(P=0.0002)$ of in-hospital mortality via a logistic model. The receiver-operating characteristic (ROC) curve for NLCR predicting in-hospital mortality showed an area under the curve (AUC) of 0.8007 which is an indicative of high predictive power. Its sensitivity and specificity is calculated to be $97.37 \%$ and 93.18 , respectively. Overall, the performance of NCLR as a predictor based on its computed hit rate is found to be approximately $93.33 \%$.Conclusion: Increased NLCR levels were independently associated with unfavorable clinical prognosis in patients with sepsis. A high NLCR $(>10)$ is significantly associated with in- hospital mortality. NCLR predicts in-hospital mortality with high accuracy, high precision and small misclassification.

Key words: Sepsis, Septic shock, Neutrophil-Lymphocyte count ratio, Hospital mortality, Rizal medical center

\section{INTRODUCTION}

Sepsis and septic shock is becoming the leading cause of morbidity and mortality in both developed and developing countries like Philippines. A recent analysis of National Surgical Quality Improvement Project Database indicated that sepsis and septic shock were ten time more common that pulmonary embolism and myocardial infarction. Several biomarkers such as Procalcitonin and C-reative protein have been historically used as indicator of bacterial infection in sepsis and septic shock with very limited specificity and sensitivity, remains expensive in countries with low income and is not systematically used at hospitals, placing them in practical and out of reach from poor patients. Hence, the researcher would like to evaluate Neutrophil-Lymphocyte Count Ratio (NLCR) as an indicator of in-hospital mortality and bacteremia among sepsis and septic shock patients, considering that NLCR 
is a readily accessible biomarker, a lot cheaper and can be calculated based on a complete blood count.

A prospective, observational study ${ }^{1}$ conducted by Hota et. al indicated that NLCR was found to be $86.2 \%$ sensitivity and $85.7 \%$ specificity, positive predictive value of $89.2 \%$ in predicting diagnosis and prognosis of sepsis which established that NLCR is a simple and effective prognostic marker of sepsis with low cost. Meanwhile, a study conducted by Ljungstrom ${ }^{2}$ et. al showed NLCR performs better than PCT as a biomarker for bacteremia and severe sepsis in the emergency department. In this study, NCLR has significantly higher sensitivity than PCT at recommended cutoff levels for bacteremia.

NLCR can help discriminate Systemic Inflammatory Response Syndrome (SIRS) due to sepsis and could predict bacteremia. Early recognition of sepsis and prompt initiation of antibiotics is of utmost importance. Septic patients during admission may be unrecognized and awaiting blood culture results may take up to one week. Utilization of an inexpensive, readily available parameter for sepsis on admission like the NLCR may improve clinical outcomes and decrease morbidity and mortality associated with delayed management. Considering that NLCR is a readily accessible biomarker, a lot cheaper and can be calculated based on a complete blood count, this crosssectional, retrospective study aims to evaluate NLCR as an indicator of in-hospital mortality and bacteremia among Sepsis and Septic Shock patients.

\section{MATERIALS AND METHODS}

This cross-sectional retrospective study is based on patients admitted in ICU or IMCU at Rizal Medical Center study from January 1, 2015 to December 30, 2016 with the diagnosis of Sepsis and Septic shock patients. Clinical assessments by attending resident physician with blood culture, laboratory and radiologic findings were used for defining the criteria for admission. Diagnosis was also established based on clinical presentations, physical examinations and laboratory investigations. All patients with age at least 18 years old diagnosed with sepsis due to one of following infections: community acquired pneumonia, hospital-acquired pneumonia, ventilator associated pneumonia, urinary tract infection, pyelonephritis, intra-abdominal infection or primary bacteremia and whose blood sampling occurred within 24 hours from presentation of sepsis are included in this study. Patients with hematologic disease, HIV infection, recently or presently receiving chemotherapy or glucocorticoids, and with contaminated blood cultures were excluded in this study. Patients with incomplete data were also not considered. Sepsis was diagnosed on the presence of SIRS that has a proven or suspected microbial etiology while Septic Shock was defined as the sepsis with hypotension (arterial blood pressure $<90 \mathrm{mmHg}$ systolic, or $40 \mathrm{mmHg}$ less than patient's normal blood pressure) for at least 1 hour despite adequate fluid resuscitation; or need for vasopressor to maintain systolic blood pressure $\geq 90 \mathrm{mmHg}$ or mean arterial pressure $\geq 70 \mathrm{mmHg}$. Clinical data of all the patients were retrieved and documented from the medical records section and Department of Laboratory Medicine. All subjects were subjected to detailed history, physical examinations and relevant investigations. Venous sample was collected for routine hematological testing in $3 \mathrm{ml} \mathrm{BD}$ vacutainer containing K3 EDTA $5.4 \mathrm{mg}$ (complete blood count).Blood cultures were extracted upon admission when clinically indicated. All blood cultures were incubated for five days and all isolates were identified by standard microbiologic procedures. Cultures from suspected source of infection such as urine, sputum, wound, endotracheal, pleural fluid and peritoneal fluid were also examined. Baseline complete blood count (CBC) was extracted during admission.WBC and NLCR were determined using the Sysmex XN-2000 automated hematology analyzer. The NLCR was calculated by dividing the neutrophil count with the lymphocyte count.

One-hundred twenty (120) patients were enrolled in this study as this is the minimum sample size requirement needed to provide $80 \%$ power of test with maximum allowable error of $5 \%$.A cut-off of at least 10 was shown to be a marker for bacteremia which was based on the previous sensitivity of NLCR $<=10$ predicting positive blood culture of $89.66 \%$ and prevalence of positive blood culture of $46 \%$. Hence, all patients were stratified into two groups, NLCR $<=10$ and NLCR $>10$.

Descriptive statistics such as counts, summary statistics and percentages were computed to describe all patients included in the study. Chi-Square Test for Independence was utilized to determine the existence of significant association between NLRC and in-hospital mortality. Meanwhile, Binary Logistic Regression Model, Odds Ratio and Receiver operating characteristic (ROC) curves were used to assess the performance of NLCR in predicting in-hospital mortality. Sensitivities and specificities were also calculated to determine the overall predictive power of NLCR as prognostic marker of bacteremia.

\section{RESULTS}

A total of 120 patients were included in this study. Out of 120,78 patients (with mean age of 60 ) has NLCR of at least 10 while only 42 patients (with mean age of 55) has NCLR of at most 10. As shown in Table 1, a high percentage of females are observed in both groups which means that majority of the 


\begin{tabular}{|c|c|c|c|}
\hline Parameter & $\begin{array}{l}\text { NLCR>10 } \\
(n=78)\end{array}$ & $\begin{array}{l}\text { NLCR } \leq 10 \\
(n=42)\end{array}$ & $p$ value \\
\hline $\begin{array}{l}\text { Age (years), } \\
\text { Mean } \pm S d\end{array}$ & $60.3 \pm 17.5$ & $55.0 \pm 17.0$ & $0.1181^{\mathrm{ns}}$ \\
\hline \multicolumn{4}{|l|}{ Age, n,\% } \\
\hline$\leq 60$ & $36(46.2)$ & 25 (59.5) & \multirow[t]{2}{*}{0.1641} \\
\hline$>60$ & $42(53.8)$ & $17(40.5)$ & \\
\hline \multicolumn{4}{|l|}{ Gender, n,\% } \\
\hline Male & $34(43.6)$ & $20(47.6)$ & \multirow[t]{2}{*}{$0.6735^{\text {ns }}$} \\
\hline Female & $44(56.4)$ & $22(52.4)$ & \\
\hline \multicolumn{4}{|l|}{ Co-morbidities, n,\% } \\
\hline Hypertension & $37(47.4)$ & $15(35.7)$ & 0.2184 ns \\
\hline Diabetes mellitus & $16(20.5)$ & $10(23.8)$ & $0.7083^{n s}$ \\
\hline Cancer & $3(3.8)$ & $3(7.1)$ & $0.4288^{n s}$ \\
\hline Kidney disease & $7(9.0)$ & $3(7.1)$ & $0.7206^{n s}$ \\
\hline COPD & $3(3.8)$ & $1(2.4)$ & 0.6839 ns \\
\hline Heart disease & $10(12.8)$ & $3(7.1)$ & 0.3394 ns \\
\hline Liver disease & $1(1.3)$ & $0(0.0)$ & 0.4599 ns \\
\hline Pulmonary & $25(32.9)$ & $10(25.0)$ & $0.3274^{\mathrm{ns}}$ \\
\hline \multicolumn{4}{|l|}{$\begin{array}{l}\text { Source of } \\
\text { bacteremia, n,\% }\end{array}$} \\
\hline Gastrointestinal & $8(10.3)$ & 5 (11.9) & 0.7890 ns \\
\hline Genitourinary & $4(5.1)$ & 5 (11.9) & $0.1786 \mathrm{~ns}$ \\
\hline Soft tissue & $6(7.7)$ & $3(7.1)$ & $0.9056^{\mathrm{ns}}$ \\
\hline
\end{tabular}

patients are females in this study. The top four comorbidities for patients with NLCR above 10 are Hypertension, Pulmonary, Diabetes Mellitus and Heart Disease. Meanwhile, the top comorbidities for patients with NLCR at most 10 are Hypertension, Diabetes Mellitus and Pulmonary.

Both groups have generally the same demographic profile in terms of proportion of above 60 years old $(\mathrm{P}=0.1641)$, gender split $(\mathrm{P}=0.6735)$ and presence of co-morbidities

such as hypertension $(\mathrm{P}=0.2184)$ and diabetes mellitus $(p=0.7083)$. Likewise, no significant difference was found on the proportion of Cancer $(p=0.4288)$, kidney disease $(p=0.7206)$, COPD $(p=0.6839)$, Heart Disease $(p=0.3394)$ pulmonary $(p=0.3274)$ and Liver Disease $(p=0.4599)$. The source of bacteria also turned out be not significantly different between the two groups. Sources considered are gastrointestinal $(\mathrm{p}=0.7890)$, genitourinary $(\mathrm{P}=0.1786)$ and soft tissue $(p=0.9056)$.

As shown in Table 2, there exists a significant association $(\mathrm{P}=0.0001)$ between NCLR and in-hospitality mortality with an estimated correlation of 0.7706 indicative of strong and positive association between the NCLR and inhospitality mortality. An increased in-hospital mortality rate is observed as the value of the NLCR increases, particularly, for patients with NLCR above 10.

The logistic model of NLCR and In-hospital mortality Table 3 showed that NLCR is a significant predictor of the latter. The odds ratio of 1.6372 indicates that the patients

\begin{tabular}{|c|c|c|c|}
\hline \multirow[t]{2}{*}{ NLCR } & \multicolumn{3}{|c|}{ In-hospital mortality } \\
\hline & No & Yes & Total \\
\hline$N L C R<=10$ & $19(15.4)$ & $23(26.6)$ & 42 \\
\hline NLCR>10 & $25(18.6)$ & $53(49.4)$ & 78 \\
\hline Total & 44 & 76 & 120 \\
\hline \multicolumn{4}{|c|}{ Test for correlation of NCLR groups and in-hospital mortality } \\
\hline \multicolumn{3}{|c|}{ Chi-square statistic } & 125.2827 \\
\hline \multicolumn{3}{|c|}{ Chi-square test $p$ value } & 0.0001 \\
\hline \multicolumn{3}{|c|}{ Fisher's exact test $p$ value } & 0.0003 \\
\hline \multicolumn{3}{|c|}{ Cramer's V correlation } & 0.7706 \\
\hline
\end{tabular}

$\begin{aligned} & \text { Table 3. Logistic model of NLCR and in-hospital } \\
& \text { mortality among sepsis and septic shock } \\
& \text { patients }\end{aligned}$
\begin{tabular}{lcc} 
Parameter & Odds ratio estimates & P-value \\
\hline Intercept & 0.849 & 0.0001 \\
NLCR & 1.6372 & 0.0002 \\
(above 10 vs at most 10) & & \\
$\begin{array}{l}\text { Chi-square statistic } \\
\text { p-value }\end{array}$ & & 127.71 \\
\hline
\end{tabular}

with NLCR above 10 are more prone to suffer with inhospital mortality. In particular, the odds of suffering in-hospital mortality of patients with NLCR above 10 is higher than the odds of those patients with NLCR at most 10 by approximately $63.7 \%$.

NCLR also showed high sensitivity (97.37\%), specificity $(93.18 \%)$ and hit rate $(93.33 \%)$ values which is an indication that NCLR is an effective and good predictor of in-hospital mortality among Sepsis and Septic Shock patients.

The area under the curve Figure 1 is also found to be 0.8007 which is an indicative of high predictive power and hence, a good indication of accuracy. The concordant index of the logistic model gives consistent results with the value of 0.797 . Hence, NLCR predicts in-hospital mortality with high accuracy and small misclassification rate.

Moreover, the sensitivity of NLCR in predicting positive culture is only $68 \%$ while specificity is around $38.6 \%$. The predictive value of NLCR for positive culture is 64.5 while for negative is 42.5 . The area under the curve of NLCR in predicting positive blood culture is 0.533 which is indicative of moderately high predictive power.

\section{DISCUSSION}

Culture of microorganisms is the gold standard for confirming bacterial infection. However, it is timeconsuming in nature and is too expensive. Currently, 


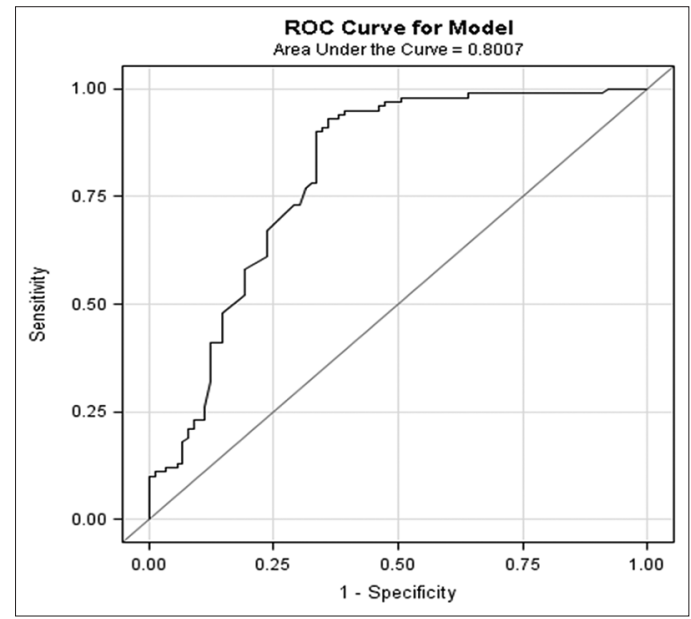

Figure 1: ROC CURVE of the NLCR logistic model

the conventional infection markers as WBC count and CRP have relatively poor discriminatory capacity in distinguishing bacterial versus non-bacterial infections. Procalcitonin, a new biomarker, has a higher predictive value for septicemia but is not readily available in most hospitals in the Philippines and is expensive.

In search for other infection markers, the NLCR which is computed by dividing neutrophil and lymphocyte count is a readily available parameter and may provide additional diagnostic value with no additional costs in septic patients. Although the available information is still far from sufficient to comprehend thoroughly the economic burden of sepsis on an international scale, current studies demonstrate that sepsis has been a serious public health problem. The patients with Septic shock have high risk of death, complications, and resource utilization Undoubtedly, the pivotal measure of improving outcome is to identify the septic patients with poor prognosis accurately.

The best of our knowledge, immune competent leukocyte plays an important role in the systemic inflammatory response to infection. Most of the prognostic scores use leukocytosis (above $12.0 \times 109 / \mathrm{L}$ ) or leukopenia (below $4.0 \times 109 / \mathrm{L}$ ) as a severity index, but few consider the leukocyte subpopulations. Significant differences exist between circulating neutrophil and lymphocyte counts and, consequently, their ratio referred to as the NLCR-has been increasingly used in the prediction of the severity or prognosis in different clinical settings, including systemic inflammation and sepsis, ischemic events and cancer. The cause responsible for NLCR elevations correlating with poor outcome in patients with sepsis remains unclear, although there are a variety of possible explanations. One of the most convincing explanations is based primarily on the physiological link between neutrophilia and lymphopenia with systemic inflammation and stress.
In this study, a total of 120 patients were included and divided into 2 groups based on the NLCR cutoff. Baseline demographics in both groups were similar. Gram positive septicemia was more common than gram- negative septicemia. Most common gram-negative organism isolated was Escherichia coli and pseudomonas accounting for $60 \%$ of the gram-negative bacteremia, and the most common gram-positive organism isolated was Streptococcus species accounting for $66 \%$ of the gram-positive bacteremia. The most common source ofinfection was respiratory tract $(32.9 \%)$, followed by genitourinary tract $(5.1 \%)$ then soft tissue infection $(7.7 \%)$.

The existence of significant association between NLCR and in-hospital mortality, which was also proven in previous studies $^{3-7}$, was found at 0.005 level of significance with correlation of 0.7706 indicative of positive and strong relationship between NLCR and in-hospital mortality. The positive sign of this correlation is a proof that that there is an increased in-hospital mortality rate as the value of the NLCR increases, particularly, for patients with NLCR above 10. The odds of suffering in-hospital mortality of patients with NLCR above 10 is 1.637 timesthan the odds of suffering in-hospital mortality of patients with NLCR at most 10. With this, the Neutrophil-lymphocyte count ratio is a good candidate of predicting the risk stratification in terms of in-hospital mortality among sepsis and septic shock patients. This finding is consistent to a prospective observational study conducted by Xuan Liu ${ }^{8}$ et al.on prognostic significance of NLCR in patients with sepsis. Clearly, the risk of death was associated with neutrophil count increase lymphocyte count decrease, and subsequently, an increase in the NLCR in the patients with sepsis at the time of admission, which was indicated in these two studies.

In this study, a logistic model was created to explore further the association of NLCR and in-hospital mortality. Results showed that NLCR is a significant predictor and the odds of suffering in-hospital mortality are higher to those patients with NLCR above 10.

Favorable results were also obtained in accessing the performance of NCLR in predicting in-hospital mortality. The probability that the NLCR correctly classified patients who suffered in-hospital mortality was found to be $97.37 \%$ and the probability that the NLCR correctly classified patients who did not suffer in-hospital mortality was computed to be $93.18 \%$. The overall performance of NCLR as a predictor of in-hospital mortality is found to be 93.33\%. These findings confirmed the conclusion obtained from the study conducted by Yong $\mathrm{Xia}^{9}$ et.al which showed that NLCR a good prognostic marker among sepsis patients as indicated by moderately high to high sensitivity and specificity values. 
A moderately high predictive power of NLCR as prognostic marker of positive blood culture is observed which is a similar finding from a studies ${ }^{10,11}$ that concluded that in an emergency care setting, NLCR is better predictor of bacteremia than routine parameters like CRP level, WBC count and neutrophil count.

\section{CONCLUSION}

Usefulness of Neutrophil Lymphocyte count ratioin risk stratification in Sepsis and Septic Shock patients was determined as NLCR is showed to be a good predictor of bacteremia and in-hospital mortality with high accuracy, significant classification rate and positively strong correlation. Patients with bacteremia and persistently high neutrophil lymphocyte have a significantly increased risk of mortality. Consequently, those patients with NLCR above 10 have higher risk of in-hospital mortality. Favorable results were also obtained in accessing the performance of NCLR in predicting in-hospital mortality.

This marker is simple, easily obtained and calculated, and in easy to integrate in daily practice without extra costs.In our setting, this simple and practical laboratory test may be utilized as one of the bases to stratify patients with sepsis syndrome, in lieu of the available but more expensive tests (e.g. Procalcitonin and CRP). It is a parameter that a clinician in an acute care and critical care setting may find useful in his or her choice of antibiotic regimen, further diagnostic examinations and choice of accommodation knowing the risk of mortality of his patients.

\section{RECOMMENDATIONS}

The retrospective study design is prone to bias, and we are unable to adjust for risk factors other than sex and age in the cox regression model. As with any retrospective review there are limitations in data available, a prospective Randomized Control Trails with further additional studies with larger population group to investigate the role of Neutrophil Lymphocyte as a prognostic factor in the severity of sepsis and its usefulness as a basis in modifying treatment. We have to look into the other possible cause of mortalities, other than infection.

\section{REFERENCES}

1. Hota PK and Reddy BG. Role of eosinophil count and neutrophil: lymphocyte count ratio as prognostic markers in patients with sepsis. Int Surg J 2017; 4:2243-2246.

2. Ljungström L, Pernestig AK, Jacobsson G, Andersson R, Usener B and Tilevik D. Diagnostic accuracy of procalcitonin, neutrophil-lymphocyte count ratio, C-reactive protein, and lactate in patients with suspected bacterial sepsis. PLoS ONE 2017; 12(7): e0181704.

3. Sarraf KM, Belcher E, Raevsky E, Nicholson AG, Goldstraw P, Lim E, et al. Neutrophil/lymphocyte ratio and its association with survival after complete resection in non-small cell lung cancer. Journal of Thoracic Cardiovascular Surgery 2009; 137: 425-428.

4. Salciccioli JD, Marshall DC, Pimentel MAF, Santos MD, Pollard T, Celi LA, et al. The Association between the Neutrophilto-Lymphocyte Ratio and Mortality in Critical IIIness: An Observational Cohort Study, Critical Care 2015; 19:13.

5. Hong DY, Kim JW, Paik JH, Jung HM, Baek KJ, Park SO, et al. Value of plasma neutrophilgelatinase-associated lipocalin in predicting themortalityof patients with sepsis at the emergency department. Clinica Chimica Acta 2016; 452:177-181.

6. Riché F, Gayat $E$, Barthélémy R, Le Dorze $M$, Matéo J and Payen D. Reversal of neutrophil-to-lymphocyte count ratio in early versus late death from septic shock. Critical Care 2015; 19: 439.

7. Terradas R, Grau S, Blanch J, Riu M, Saballs P and Castells X. Eosinophil Count and Neutrophil-Lymphocyte Count Ratio as Prognostic Markers in Patients with Bacteremia: A Retrospective Cohort Study. PLoS ONE 2012; 7(8): e42860.

8. Liu X, Shen $\mathrm{Y}$, Wang $\mathrm{H}, \mathrm{Ge} \mathrm{Q}$, Fei A and Pan S. Prognostic Significance of Neutrophil-to-Lymphocyte Ratio in Patients with Sepsis: A Prospective Observational Study. Mediators of Inflammation 2016; 8191254.

9. Xia Y, Xu-Guang Guo, Tian-Xing Ji and Chen Q. Neutrophil Count to Lymphocyte Ratio is a Potential Diagnostic Index for Bacteremia in Adult. Life Science Journal 2014; (11)1.

10. De Jager CP, van Wijk PT, Mathoera RB, de Jongh-Leuvenink J, van der Poll T and Wever PC. Lymphocytopenia and neutrophillymphocyte count ratio predict bacteremia better than conventional infection markers in an emergency care unit. Critical Care 2010; 14(5): R192.

11. Bates DW, Pruess KE and Lee TH. How bad are bacteremia and sepsis? Outcomes in a cohort with suspected bacteremia. Arch Intern Med 1995; 155:593-598.

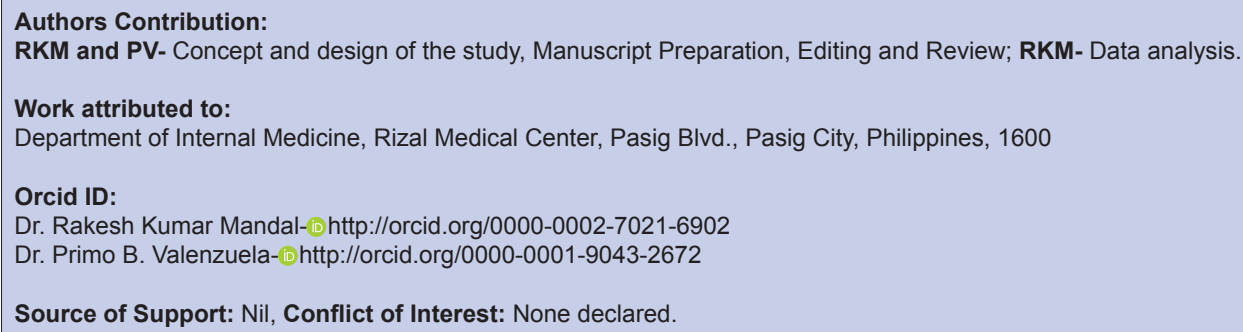

